\title{
Mitochondrial DNA disturbances and deregulated expression of oxidative phosphorylation and mitochondrial fusion proteins in sporadic inclusion body myositis
}

Marc Catalán-García*, Glòria Garrabou*, Constanza Morén*, Mariona Guitart-Mampel*, Adriana Hernando*, Àngels Díaz-Ramos $† £ \S$, Ingrid González-Casacuberta*, Diana-Luz Juárez*, Maria Bañó*, Jennifer Enrich-Bengoa*, Sonia Emperador\|, José César Milisenda*, Pedro Moreno*, Ester Tobías*, Antonio Zorzano†キ£, Julio Montoya\|, Francesc Cardellach* and Josep Maria Grau*

*Muscle Research and Mitochondrial Function Laboratory, CELLEX-IDIBAPS, Faculty of Medicine, University of Barcelona; Internal Medicine Department, Hospital Clinic of Barcelona, CIBERER-U722, Barcelona, Spain

†Institute for Research in Biomedicine (IRB Barcelona), Barcelona Institute of Science and Technology, Barcelona, Spain †Departament de Bioquímica i Biomedicina Molecular, Facultat de Biologia, Universitat de Barcelona, 08028 Barcelona, Spain $\S$ CIBER de Diabetes y Enfermedades Metabólicas Asociadas (CIBERDEM), Instituto de Salud Carlos III, Madrid, Spain ॥Departamento de Bioquímica, Biología Molecular y Celular, Universidad de Zaragoza, CIBERER-U727, Instituto de Investigaciones Sanitarias de Aragón, Zaragoza, Spain

\begin{abstract}
Sporadic inclusion body myositis (SIBM) is one of the most common myopathies in elderly people. Mitochondrial abnormalities at the histological level are present in these patients. We hypothesize that mitochondrial dysfunction may play a role in disease aetiology. We took the following measurements of muscle and peripheral blood mononuclear cells (PBMCs) from 30 sIBM patients and 38 age- and gender-paired controls: mitochondrial DNA (mtDNA) deletions, amount of mtDNA and mtRNA, mitochondrial protein synthesis, mitochondrial respiratory chain (MRC) complex I and IV enzymatic activity, mitochondrial mass, oxidative stress and mitochondrial dynamics (mitofusin 2 and optic atrophy 1 levels). Depletion of mtDNA was present in muscle from sIBM patients and PBMCs showed deregulated expression of mitochondrial proteins in oxidative phosphorylation. MRC complex IV/citrate synthase activity was significantly decreased in both tissues and mitochondrial dynamics were affected in muscle. Depletion of mtDNA was significantly more severe in patients with mtDNA deletions, which also presented deregulation of mitochondrial fusion proteins. Imbalance in mitochondrial dynamics in muscle was associated with increased mitochondrial genetic disturbances (both depletion and deletions), demonstrating that proper mitochondrial turnover is essential for mitochondrial homoeostasis and muscle function in these patients.
\end{abstract}

Key words: deletions, depletion, mitochondria, mitofusin-2, OPA1, sporadic inclusion body myositis.

\section{INTRODUCTION}

Sporadic inclusion body myositis (sIBM) is the most common inflammatory myopathy in individuals aged $>50$ years [1] and one of the most important myopathies associated with ageing [2]. With a male:female ratio of 3:1, it is a rare disease (ORPHA611) and its prevalence varies from 4.7 per million in the Netherlands to 14.9 per million in western Australia [3]. These rates continue to increase, probably due to improved diagnostic protocols and increased ageing of the population. This myopathy is a devastating condition, causing slow, progressive muscle weakness and wasting, with quadriceps and finger flexors being the muscles most typically affected. Nevertheless, the clinical presentation varies considerably. This muscle weakness usually leads to falls and difficulty in standing. Around $60 \%$ of the patients also present with dysphagia. With the wide spectrum of clinical

Abbreviations: $\mathrm{CK}$, creatine kinase; COX, cytochrome $c$ oxidase; CS, citrate synthase; H\&E, haematoxylin and eosin; HAE, hydroxyalkenal; IBMFRS, Inclusion Body Myositis Functional Rating Scale; MDA, malondialdehyde; MFN2, mitofusin 2; MRC, mitochondrial respiratory chain; NSE, non-specific esterase; OPA1, optic atrophy 1; ORO, Oil Red O; PAS-D, periodic acid-Schiff diastase stain; PBMC, peripheral blood mononuclear cell; RT-PCR, reverse transcriptase PCR; ROS, reactive oxygen species; SDH, succinate dehydrogenase; sIBM, sporadic inclusion body myositis; TRC, tyrosine-rich crystalloids; VDAC1, voltage-dependent anion channel 1. 
manifestations in sIBM, muscle biopsy continues to be the gold standard for diagnosis [4].

The histological features observed in muscle biopsies of sIBM patients include: (i) inflammatory changes with predominant CD8+ T-cell infiltrates and the expression of MHC-I antigens by non-necrotic muscle fibres, (ii) different degrees of degenerative changes in muscle fibres and the presence of rimmed vacuoles composed mainly of $\beta$-amyloid, phosphorylated tau and caveolin proteins, among others, and (iii) mitochondrial abnormalities characterized by the presence of ragged-red fibres, cytochrome $c$ oxidase (COX)-negative and succinate dehydrogenase (SDH)positive muscle cells [5,6], all of which are widely associated with mitochondrial dysfunction and ageing $[1,2,7]$. However, the distribution of these histological features is not homogeneous, and they may not be simultaneously present, particularly in the early stages of the disease. For all these reasons, and due to its slow progression, diagnosis may be delayed for 5-10 years.

Mitochondria are the cell's powerhouse. They are responsible for most of the energy supply, metabolic reactions, calcium homoeostasis and cell respiration. However, under pathological conditions, mitochondria are the main centres for reactive oxygen species (ROS) production and for triggering apoptosis. Mitochondria are essential for cell bioenergetics, especially in highly energetic tissues such as muscle, in which mitochondrial dysfunction may become evident much earlier. Molecular mitochondrial alterations have recently been found in SIBM by down-regulated expression of mitochondrial respiratory chain (MRC) complex I subunits and mitochondrial DNA (mtDNA) variations [7]. In addition, mtDNA deletions have also been reported in SIBM patients $[6,8]$, but not correlated with abnormal mitochondrial function or dynamics. Despite mitochondrial abnormalities having classically been described at a histological level, mitochondrial dysfunction in SIBM patients has scarcely been assessed at a molecular level.

Mitochondrial dynamics is a recently discovered mechanism responsible for mitochondrial turnover and renewal [9]. Mitochondria constitute a complex network that is constantly undergoing fusion and fission processes to exchange genetic and structural components, which include mtDNA and MRC machinery. Deregulation of mitochondrial dynamics has been associated with disease, but has not previously been described in SIBM patients [10-16].

Although the pathological features of sIBM have been widely described, its aetiology remains unknown. It has been proposed that its development could be due to a complex interaction of environmental agents, accelerated ageing and genetic susceptibility $[2,17]$. As muscle tissue has a high dependence on ATP to exert its function, mitochondrial alterations in SIBM could be one of the factors involved in triggering muscle weakness and degeneration, because these alterations have been related to muscle disease by many authors [18-20]. For an in-depth evaluation of the pathological characteristics of the disease, we describe the mitochondrial phenotype at a genetic, molecular and functional level in sIBM patients.

To determine potential disease biomarkers we assessed abnormal mitochondrial fingerprints, in the target tissue of the disease (muscle) and with less invasive approaches using peri- pheral blood mononuclear cells (PBMCs). This PBMC model is widely feasible for the evaluation of mitochondrial function [21-23]. Thus, we designed the present study to evaluate mitochondrial dysfunction in both muscle and PBMCs of sIBM patients to correlate dysfunction severity with genetic and molecular mitochondrial alterations, and determine their potential association with the deregulation of mitochondrial dynamics.

\section{MATERIALS AND METHODS}

\section{Study design}

We performed a single-site, cross-sectional, case-control, observational study.

\section{Study population, diagnosis, clinical data and sample collection}

A total of 30 patients with sIBM who had attended the Internal Medicine Department of the Hospital Clinic of Barcelona (Spain) over the last 20 years were prospectively and consecutively included. These patients were age and gender paired with 38 controls. All muscle biopsies were performed for diagnostic purposes indicated for muscle weakness or raised creatine kinase (CK) levels. Surgical muscle biopsies were obtained by trained physicians, and the samples were processed routinely in the laboratory as described elsewhere [24]. For histological studies fresh muscle samples were frozen in cooled isopentane, sectioned by cryotome at $-30{ }^{\circ} \mathrm{C}$ and stained with different reagents for diagnostic purposes: haematoxylin and eosin (H\&E), tyrosine-rich crystalloids (TRCs), non-specific esterase (NSE), periodic acid-Schiff diastase stain (PAS-D), Oil Red O (ORO), acid and alkaline phosphatase, NADH, COX, SDH and ATPase at pH 4.3, 4.6 and 9.4, as reported elsewhere [24]. In addition some immunohistochemistry reactions such as class I antigens from the MHC as well as p62 were performed. The same expert pathologist (J.M.G.) read all the samples. Leftover biopsy material from both sIBM patients and individuals with no histological myopathy was included as patient and control samples, respectively. Histological data were collected to study inflammation and the presence of ragged-red fibres, COX-negative and SDH-positive fibres, as well as the number of vacuolated muscle cells. Diagnosis of sIBM was considered as definite or probable according to the criteria of the European Neuromuscular Centre [1,25]. Samples from 23 muscle biopsies from sIBM patients and 18 from controls were included following these criteria. In parallel, blood samples were collected from 14 sIBM patients and 20 control individuals free of muscle disease. Exclusion criteria were: age $<40$ years, muscle disease in the case of controls, mitochondrial disease, and family history of hereditary mitochondrial pathology, HIV infection or drug abuse. All individuals were informed, and signed written consent was obtained for inclusion in this study, which was approved by the Ethical Committee of our hospital, following the Declaration of Helsinki. All sIBM patients answered the Inclusion Body Myositis Functional Rating Scale (IBMFRS) test, which is a validated, disease-specific test to assess disease severity. 
A database was created to collect epidemiological, clinical and histological data, which were further complemented with experimental results.

Leftover samples from mandatory muscle biopsy diagnosis were included in optimal cutting temperature compound (OCT) and immediately frozen and stored at $-80^{\circ} \mathrm{C}$ until homogenization $(5 \% \mathrm{w} / \mathrm{v})$ with mannitol to perform experimental studies.

Around $20 \mathrm{ml}$ of peripheral blood was also obtained by antecubital vein puncture and collected in EDTA tubes. PBMCs were isolated using Ficoll density gradient centrifugation, divided into aliquots and stored at $-80^{\circ} \mathrm{C}$ until analysis.

\section{Mitochondrial genetic studies}

Total DNA from muscle biopsies and PBMCs was obtained by the standard phenol-chloroform extraction procedure. The assessment of mtDNA deletions was performed in muscle samples by long-range PCR using Phusion High-Fidelity PCR Master Mix with GC Buffer (F-532L, ThermoFisher Scientific) and the following primers: forward 5'-TTAGCAAGGGAACTACTCCCA-3' and reverse 5'-CGGATACAGTTCACTTTAGCTACCCCCAAGTG-3'. The methodology of this procedure was performed as previously described [26-28]. The PCR products were electrophoresed in a $0.8 \%$ agarose gel with ethidium bromide to detect different sizes in the mitochondrial genome (see Supplementary Figure S1).

To evaluate mtDNA content, fragments of the mitochondrially encoded $12 \mathrm{~S}$ rRNA gene and the nuclear-encoded RNase-P gene were amplified separately in triplicate by quantitative reverse transcriptase PCR (RT-PCR) using Applied Biosystems technology [29]. The relative mtDNA content was expressed as the ratio mtDNA 12S rRNA:nDNA RNase-P.

\section{Mitochondrial transcript quantification}

To evaluate mtRNA content in both muscle and PBMCs, total RNA was first extracted using the TriPure procedure and immediately retro-transcribed into cDNA using random hexamer primers, following previously described methodology [30]. The mtRNA:nRNA content was then measured by RT-PCR as previously described for mtDNA and expressed in the same units (mtRNA 12S rRNA:nRNA RNase-P).

\section{Mitochondrial protein quantification}

Mitochondrial protein quantification was assessed in muscle and PBMCs by the quantification of two mitochondrial complex IV protein subunits: COX-II (encoded by the mitochondrial genome) and COX-IV (encoded by the nuclear genome). These proteins were assessed through immunoblotting using SDS/7/13\% PAGE and immunodetection using previously described reagents and antibodies [22]. Mitochondrial values normalized to nuclear-encoded COX proteins were expressed as the COX-II:COX-IV ratio, and absolute values of COX-II and COX-IV were normalized with a loading control ( $\alpha$-tubulin for muscle samples and $\beta$-actin for PBMC samples) and expressed as the COX-II: $\alpha$-tubulin and COX-IV: $\alpha$-tubulin ratios in muscle or the COX-II: $\beta$-actin and COX-IV: $\beta$-actin ratios in PBMCs. COX-II and COX-IV values were also normalized by mitochondrial mass [through constitutive voltage-dependent anion channel
1 (VDAC1) expression] and expressed as COX-II:VDAC1 and COXIV:VDAC1 ratios in both tissues (see Supplementary Figure S2) [31].

\section{MRC complex I and complex IV enzyme activity}

MRC complex I (EC 1.6.5.3) and IV (COX - EC 1.9.3.1) enzyme activities were measured spectrophotometrically according to the methodologies of Spinazzi et al. [32] in both muscle homogenates and PBMCs. Specific enzymatic activities were expressed in absolute values as nanomoles of substrate consumed or product generated per minute per milligram of cell protein $(\mathrm{nmol} / \mathrm{min}$ per $\mathrm{mg}$ protein) or in relative values with respect to mitochondrial content normalized by citrate synthase (CS) activity (COX:CS).

\section{MRC complex assembly}

Total oxidative phosphorylation rodent WB Antibody Cocktail (MS604, Abcam) was used to detect one subunit from each MRC complex (I-V): NDFUFB8 from complex I, SDHB from complex II, UQCRC2 from complex III, MTCO1 from complex IV and ATP5A from complex V. These subunits are labile when the respiratory complex to which they belong is not assembled and a decrease in this subunit would manifest this phenomenon. To normalize the levels of expression of these five proteins from the MRC we analysed using the SYPRO technique for the total protein content and the VDAC1 levels for the mitochondrial mass in the same membrane.

\section{Mitochondrial mass analysis}

Mitochondrial mass was analysed in both muscle and PBMCs by spectrophotometric measurement of CS (EC 4.1.3.7) enzyme activity and, in parallel, by the measurement of VDAC1 protein levels by Western blotting. CS is a mitochondrial enzyme of the citrate cycle widely considered to be a reliable marker of mitochondrial content, and VDAC1 [22] is an outer membrane anion channel also considered to be a mitochondrial mass marker [22]. CS activity was expressed as nmol/min per $\mathrm{mg}$ of protein and VDAC1 expression was normalized by $\alpha$-tubulin in muscle and by $\beta$-actin in PBMCs, both of which are validated proteins for assessing total cell loading mass and expressed as the VDAC $1: \alpha$-tubulin ratio in muscle or the VDAC- $1: \beta$-actin ratio in PBMCs.

\section{Oxidative stress assay}

Lipid peroxidation (an indicator of oxidative damage produced by ROS in cellular lipid compounds) was quantified in muscle and PBMCs using the Oxys Research kit (Deltaclon) through the spectrophotometric measurement of malondialdehyde (MDA) and 4-hydroxyalkenal (HAE), both products of fatty acid peroxide decomposition and normalized by protein content $(\mu \mathrm{M}$ MDA + HAE per mg of protein) [33].

\section{Expression of mitochondrial fusion proteins}

Mitochondrial dynamics were assessed in muscle tissue by the evaluation of both optic atrophy 1 (OPA1) and mitofusin 2 (MFN2) expression at two different levels: transcript and protein quantification. For the assessment of transcript levels of OPA1 and MFN2, mRNA, total RNA extracted by TriPure was reverse 
transcribed with the SuperScript III reverse transcriptase kit (Invitrogen). Quantitative PCRs were performed with ABI Prism 7900HT real-time PCR equipment (Applied Biosystems) using materials and reagents previously described [34,35]. All measurements were normalized to glyceraldehyde-3-phosphate dehydrogenase (GAPDH) mRNA content. Protein levels of OPA1 and MFN2 were assessed in parallel using Western blotting on SDS/7/8\% PAGE and posterior immunodetection using a BD antibody (612606) and an Abcam antibody (ab56889), respectively. In the case of OPA1, results were expressed as the ratio between the long and the short forms of the protein (OPA1 long:short), because this ratio decreases in abnormal mitochondrial dynamic states. In the case of MFN2, absolute values were normalized by total cell loading mass and expressed as the ratio MFN2: $\alpha$ tubulin.

\section{Statistical analysis}

Statistical analysis was performed using the SPSS, version 20.00 to search for differences depending on group assignment (patients vs controls or presence vs absence of mtDNA deletions) using the non-parametric Mann-Whitney U-test for independent samples. Results were expressed as means \pm S.E.M.S or as a percentage of increase/decrease with respect to controls that were arbitrarily assigned as $0 \%$. In all cases, $P<0.05$ was considered statistically significant.

\section{RESULTS}

\section{Clinical and histological data}

According to the design of the study, no statistical differences were observed with regard to age and gender between sIBM patients and controls. Clinical, epidemiological, histological and mtDNA deletion data of both patients and controls are summarized in Table 1 and histological data examples are compiled in Figure 1. At the time of study inclusion the sIBM patient cohort scored $26.61 \pm 1.19$ out of 40 with the IBMFRS test, indicating moderate-to-advanced sIBM clinical severity.

\section{MtDNA deletions}

When assessing for the presence of mtDNA deletions in muscle from sIBM patients, $57 \%$ of the sIBM cohort presented multiple mtDNA deletions, whereas the rest showed no alterations in this parameter (see Supplementary Figure S1).

\section{MtDNA content}

MtDNA content was significantly decreased by $36 \%$ in muscle from sIBM patients compared with controls $(641.6 \pm 141.2$ vs $979.7 \pm 111.3$, respectively, $P<0.05$ ) (Figure 2 ). Similarly, mtDNA was decreased, albeit not significantly, by $14.5 \%$ in PBMCs (Figure 2).

\section{Mitochondrial transcript quantification}

No variation was found in the levels of mtRNA in either muscle or PBMCs (Figure 2) of sIBM patients compared with controls.

\begin{tabular}{|c|c|c|}
\hline Muscle biopsies & sIBM $(n=23)$ & Controls $(n=18)$ \\
\hline \multicolumn{3}{|c|}{ Clinical and epidemiological data } \\
\hline Age (years) ${ }^{a}$ & $68.64 \pm 2.87$ & $63.22 \pm 2.55$ \\
\hline Male:female ratio & 0.64 & 0.38 \\
\hline IBMFRS score ${ }^{a}$ & $26.61 \pm 1.19 / 40$ & - \\
\hline mtDNA deletions $(\%)^{b}$ & 57 & - \\
\hline \multicolumn{3}{|l|}{ Histological data (\%) } \\
\hline Rimmed vacuoles ${ }^{b}$ & 61.20 & - \\
\hline Ragged-red fibres ${ }^{b}$ & 60 & - \\
\hline coX-negative fibres ${ }^{b}$ & 36.36 & - \\
\hline SDH-positive fibres ${ }^{b}$ & 44 & - \\
\hline MHC-I expression ${ }^{b}$ & 57.14 & - \\
\hline Inflammation ${ }^{b}$ & 67.74 & - \\
\hline PBMC samples & sIBM $(n=14)$ & Controls $(n=20)$ \\
\hline \multicolumn{3}{|c|}{ Clinical and epidemiological data } \\
\hline Age $\left(\right.$ years) ${ }^{a}$ & $68.43 \pm 3.28$ & $68.55 \pm 1.41$ \\
\hline Male:female ratio & 1 & 1.85 \\
\hline IBMFRS score ${ }^{a}$ & $25.85 \pm 1.31 / 40$ & - \\
\hline \multicolumn{3}{|c|}{$\begin{array}{l}{ }^{a} \text { Data presented as means }+ \text { S.E.M.s. } \\
{ }^{b} \text { Data presented as percentage. }\end{array}$} \\
\hline
\end{tabular}

\section{Mitochondrial protein quantification}

As shown in Figure 2, muscle from sIBM patients presented an increase of $25 \%$ in the COX-II:COX-IV ratio with respect to controls, suggesting increased expression of mitochondrial versus nuclear COX subunits (see Supplementary Figure S2). It is interesting that in PBMCs this ratio showed a statistically significant decrease of $45.46 \%(0.42 \pm 0.05$ vs $0.77 \pm 0.09, P<0.05$; Figure 2). In addition, in PBMCs COX-IV protein levels normalized by total cell mass showed a strong trend to be increased by $67.85 \%$ in sIBM patients compared with controls $(0.47 \pm 0.08$ vs $0.28 \pm 0.03, P=0.064$; Figure 2 ), suggesting increased nuclear versus mitochondrial COX protein expression. This abnormality became statistically significant when COX-IV values were normalized by mitochondrial mass (COX-IV:VDAC-1), which showed a $172.72 \%$ increase in sIBM patients compared with controls ( $1.80 \pm 0.35$ vs $0.66 \pm 0.01, P<0.05$; Figure 2$)$.

\section{Mitochondrial complex I and IV activity}

Measurements of respiratory chain activities showed no differences in complex I activity in muscle of patients and controls, in either absolute or relative units (data not shown). However, complex IV activity was abnormally decreased in both muscle and PBMCs of sIBM patients with respect to controls (Figure 2). Thus, taking into account absolute values, a decrease of $13.55 \%$ was found in muscle (Figure 2). These differences became statistically significant in muscle when complex IV activity was normalized to mitochondrial mass (CS), by showing a $30.31 \%$ decrease in sIBM patients compared with controls $(0.26 \pm 0.02$ vs $0.33 \pm 0.03, P<0.05$; Figure 2). On analysing this parameter in PBMCs, complex IV activity was also significantly decreased by $31.4 \%$ in absolute values in patients compared with controls $(24.32 \pm 3.1$ vs $35.45 \pm 3.4, P<0.05$; Figure 2$)$ and by $28.21 \%$ 

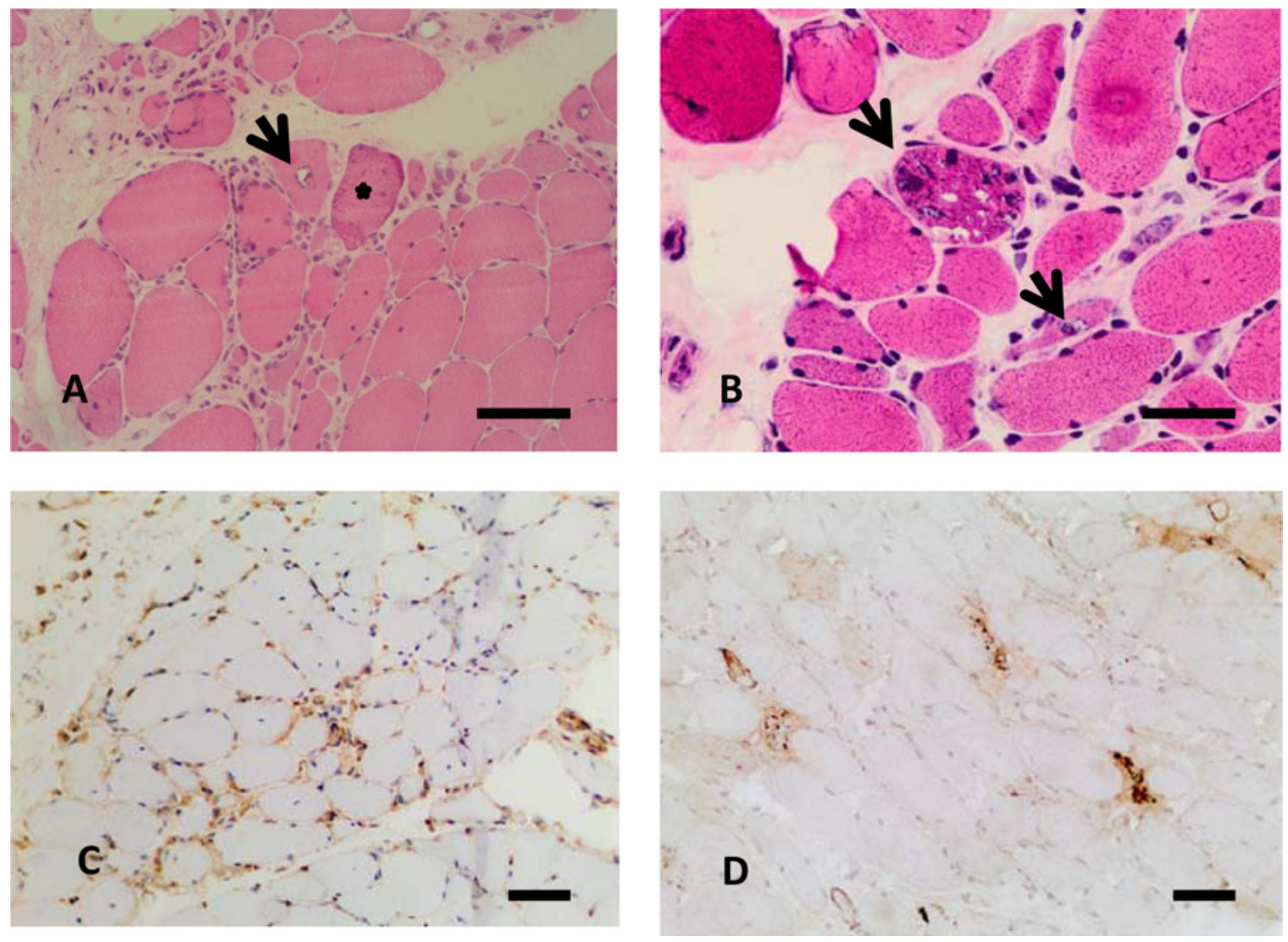

Figure 1 Images from the histological staining of sIBM muscle tissue

(A, B) H\&E in frozen muscle tissue: marked variability in fibre size. Some inflammatory cells are observed in the endomysium, together with rimmed vacuoles (arrows) and a typical ragged-red fibre (star). (C) Almost universal sarcolemmal positivity of class I antigen from the MHC, a typical feature in sIBM. (D) Positivity of p62 in rimmed vacuoles, suggestive of muscle degeneration. Scale bar $=50 \mu \mathrm{m}$.
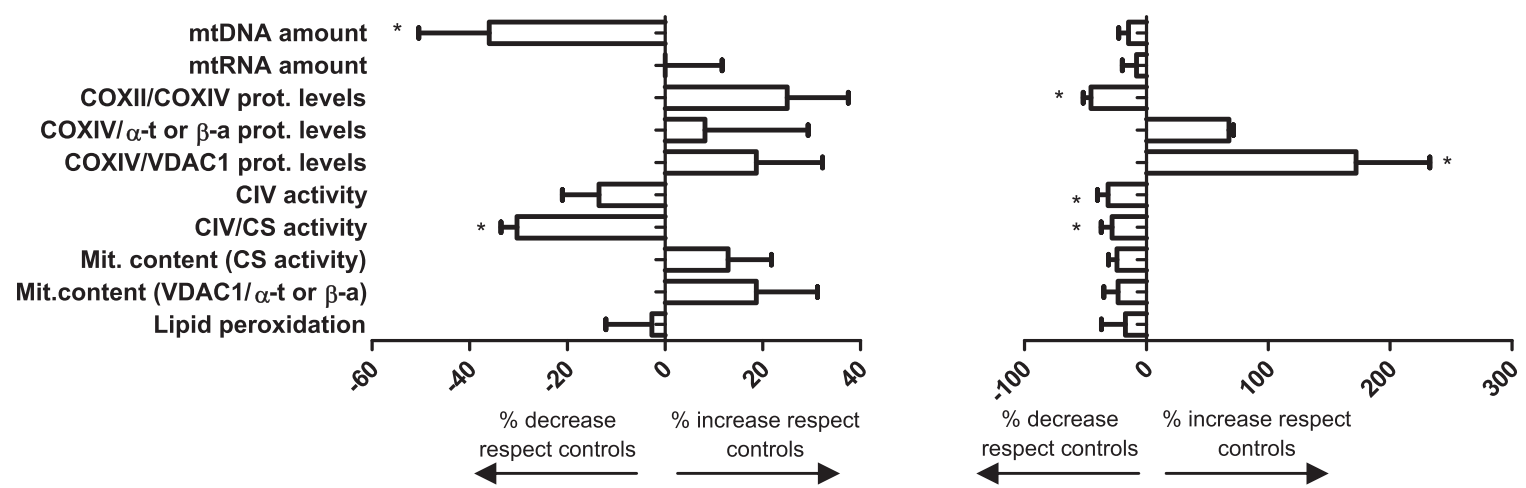

Figure 2 Mitochondrial parameters in muscle and PBMCs from sIBM patients compared with controls

Mitochondrial parameters in muscle tissue and PBMCs from patients with SIBM expressed as percentage of in crease/decrease compared with controls. A significant decrease in mtDNA amount and complex IV activity normalized to mitochondrial content can be observed in muscle, and also a significant decrease in COX-II:COX-IV ratio, complex IV absolute activity and complex IV activity normalized to mitochondrial content can be observed in PBMCs. In this muscle tissue, a significant increase in COX-IV subunit normalized to mitochondrial content can be observed as well as other non-significantly altered parameters. $\alpha$-t, $\alpha$-tubulin; $\beta$-a, $\beta$-actin; C-IV, MRC complex IV. $*: p<0.05$. 
when this parameter was normalized to the mitochondrial mass $(0.28 \pm 0.1$ vs $0.39 \pm 0.1, P<0.05$; Figure 2$)$.

\section{MRC complexes assembly}

The analysis of the subunit levels for each complex of the MRC (I-V) that correlates with the proper assembly of the respiratory chain complexes did not show differences between sIBM patients and controls, normalized to either total protein or VDAC1 content. It is interesting that the complex $\mathrm{V}$ subunit presented a trend towards a decrease in SIBM patients, but this difference did not reach statistical significance (Figure 3).

\section{Mitochondrial mass content}

Mitochondrial mass, assessed by both the quantification of CS enzyme activity and VDAC1 protein content, was increased in muscle of sIBM patients compared with controls (12.9\% in CS activity and $18.7 \%$ in VDAC1: $\alpha$-tubulin protein levels; see Figure 2) and decreased in PBMCs of SIBM patients compared with controls (by $24.18 \%$ in CS activity and $23.26 \%$ in VDAC1: $\beta$ actin protein content; see Figure 2). However, none of these results was statistically significant.

\section{Oxidative stress levels}

No differences were found in lipid peroxidation, an indicator of oxidative damage produced by ROS in lipid compounds, between sIBM patients and controls in muscle or PBMCs (see Figure 2).

\section{Quantification of mitochondrial dynamics}

Transcript levels of OPA 1 showed a significant $37 \%$ reduction in muscle of sIBM patients compared with controls $(0.59 \pm 0.11$ vs $0.94 \pm 0.19, P<0.05$; Figure 4$)$. OPA1 long:short protein isoform ratios, although not significant, were decreased by $45 \%$ in muscle of sIBM patients compared with controls $(0.55 \pm 0.17$ vs $1 \pm 0.16, P<0.083$; Figure 4$)$. In the case of MFN2 transcripts, muscle of sIBM patients showed a $31 \%$ reduction. With regard to MFN2 protein expression, a strong trend towards a decrease was observed in sIBM patients, with $54 \%$ compared with controls $(1.93 \pm 0.3$ vs $4.17 \pm 0.1, P=0.096$; Figure 4$)$. The differences in MFN2 levels in either transcript or protein content were not statistically significant.

\section{Correlation of mitochondrial parameters with regard to the presence/absence of mtDNA deletions}

Last, the sIBM cohort was divided into two groups on the basis of the presence or absence of mtDNA deletions for further exploration of mitochondrial homoeostasis, depending on this condition with respect to controls. The mtDNA content was slightly decreased in SIBM patients with mtDNA deletions compared with those without (Figure 5). These differences became statistically significant in SIBM patients with deletions with respect to the control group $(498.50 \pm 178.70$ vs $979.70 \pm 111.38, P<0.05)$. MFN2 levels showed a similar pattern to those of mtDNA content, showing the lowest content in SIBM patients with mtDNA deletions and the highest in controls $(1.16 \pm 0.24$ vs $4.17 \pm 0.96$, $P<0.05)$, with sIBM patients without mtDNA deletions presenting an intermediate value of expression (Figure 5). a)

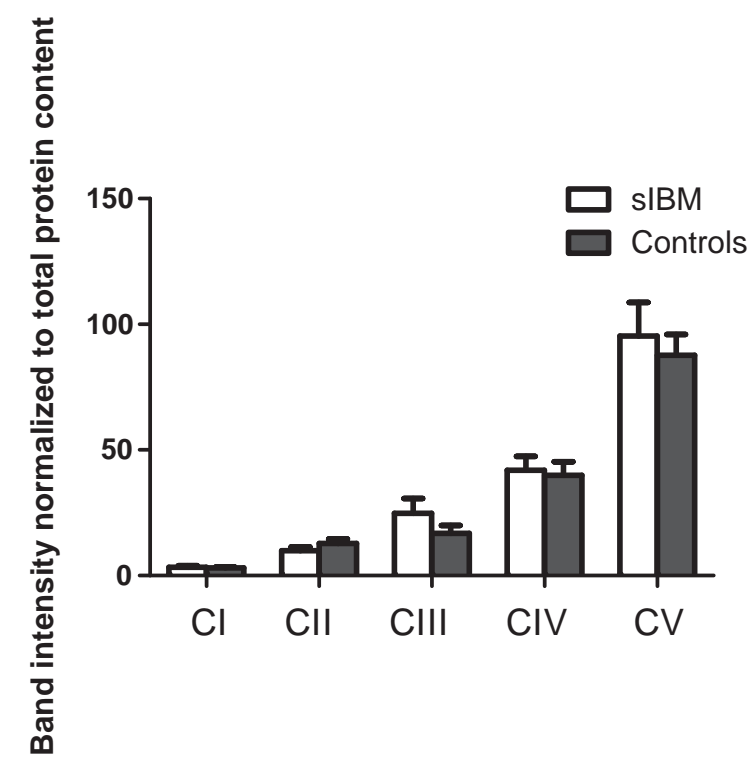

b)

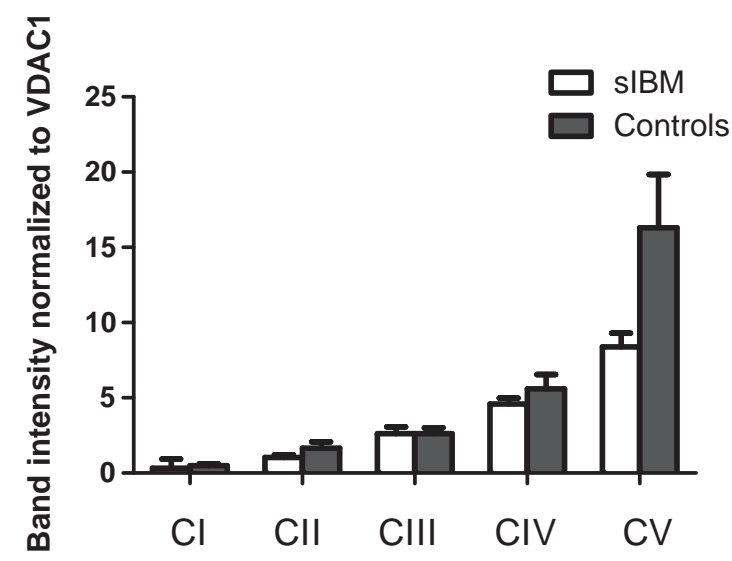

Figure 3 MRC complex assembly through the measurement of protein expression level of labile subunits of each complex in muscle tissue from SIBM patients compared with controls

No significant differences with regard to levels of specific subunits for each MRC complex were found between muscle from sIBM patients and muscle from controls, normalized to (a) total protein content and (b) the nuclear-encoded mitochondrial protein VDAC1. A trend towards increase is observed in the complex $V$ subunit when normalized to VDAC1 which, although not significant, may give us an idea of complex $\mathrm{V}$ disassembly. This multiplex Western blotting measures the protein expression level of a subunit of each MRC complex (I-V), labile when the complex is not properly assembled; the following were measured: NDFUFB8 from complex I (Cl; $20 \mathrm{kDa})$, SDHB from complex II (CII; $30 \mathrm{kDa})$, UQCRC2 from complex III (CIII; 48 kDa), MTC01 from complex IV (CIV; 40 kDa) and ATP5A from complex $\mathrm{V}(\mathrm{CV} ; 55 \mathrm{kDa})$.

\section{DISCUSSION}

Mitochondrial abnormalities in muscle from sIBM patients are accepted in the scientific community as a result of histological evidence $[7,36]$. However, little research has been performed to characterize potential functional or molecular mitochondrially 


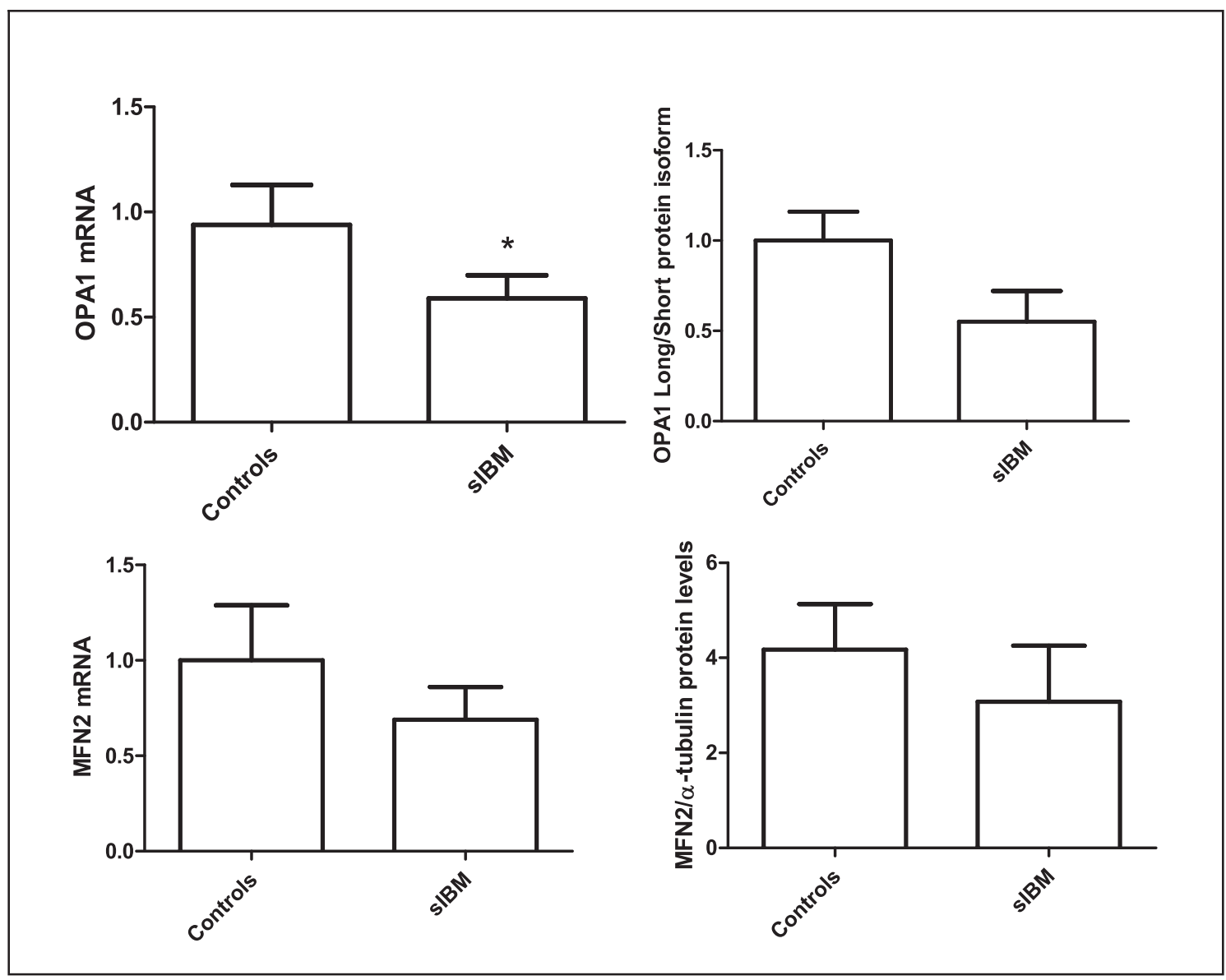

Figure 4 Mitochondrial dynamics in muscle from sIBM patients

MFN2 and OPA1 were assessed in muscle from SIBM patients at the transcript and protein levels. Significant differences were found when OPA1 mRNA levels were compared between the two groups. The other parameters showed a non-significant trend to be decreased in the sIBM respective controls. $*: p<0.05$.

disrupted pathways [6-8]. In the present study, we evaluated mitochondrial dysfunction at a genetic and molecular level, and studied its potential association with mitochondrial dynamic deregulation. We compared mitochondrial status in muscle and PBMCs from sIBM patients with that of controls, first to evaluate its involvement in disease aetiology and, second, to determine whether mitochondrial alterations are confined to muscle tissue (the target tissue of the disease) or whether these alterations extend to other body tissues, which may allow less invasive approaches and potential follow-up as a putative biomarker.

With regard to experimental data, sIBM patients showed a decrease in mtDNA content. This mtDNA depletion was statistically significant in muscle from sIBM patients, because it is post-mitotic tissue that is prone to store mitochondrial deficiencies. On the other hand, mtDNA depletion was only mildly manifested in PBMCs, probably due to their higher renewal capacity and shorter mean lifespan. Although mtDNA depletion was found in both tissues, no significant changes were observed in mtRNA levels of either muscle or PBMCs [37].

On analysing MRC function, no differences were found in complex I activity in muscle from sIBM patients compared with controls. Otherwise, MRC complex IV deficiency was present in both tissues (muscle and PBMCs) of sIBM patients. Absolute values of complex IV activity in muscle showed a trend towards a decrease, but, on normalizing these absolute values by mitochondrial mass, complex IV deficiency became significant. These phenomena may be due to the increase in mitochondrial biogenesis detected in muscle of sIBM patients as compensating for mitochondrial dysfunction. This increase in muscular mitochondrial content, characteristic of primary mitochondrial diseases [38], is observed in parallel in sIBM patients as an increase in CS activity and VDAC1: $\beta$-actin protein levels, as well as by the presence of ragged-red fibres. Similarly, mitochondrial protein synthesis tended to increase, albeit not significantly, in muscle from sIBM patients (COX-II:COX-IV), probably as a consequence of the trend of this homoeostatic muscle to increase mitochondrial biogenesis. As mentioned previously, PBMCs present a shorter mean lifespan, thereby hindering the development of homoeostatic mechanisms such as increasing mitochondrial content to deal with mitochondrial dysfunction. Thus, in PBMCs, complex IV activity was found to be significantly decreased in both absolute and relative values to mitochondrial 
a)

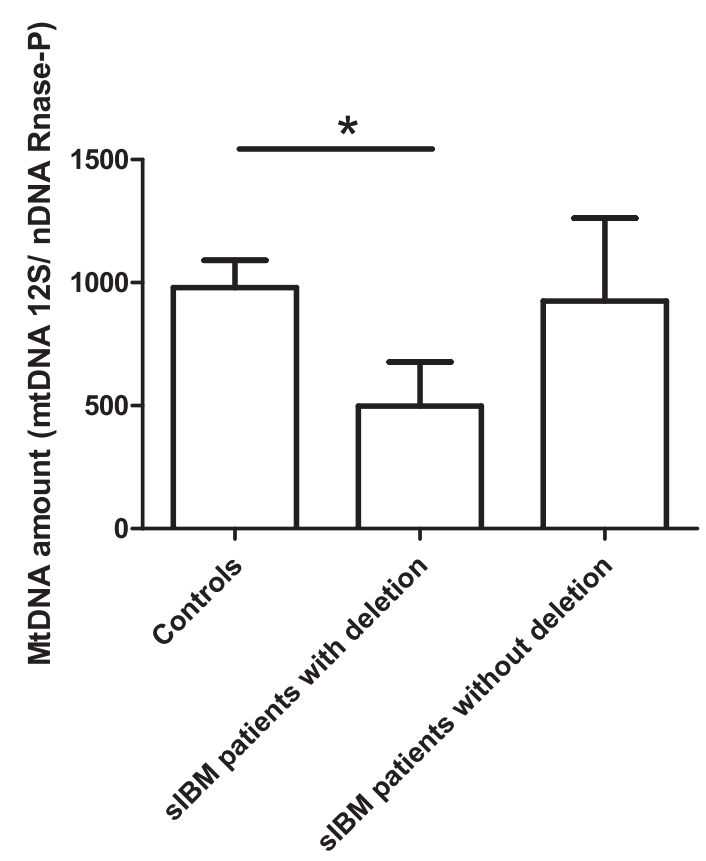

b)

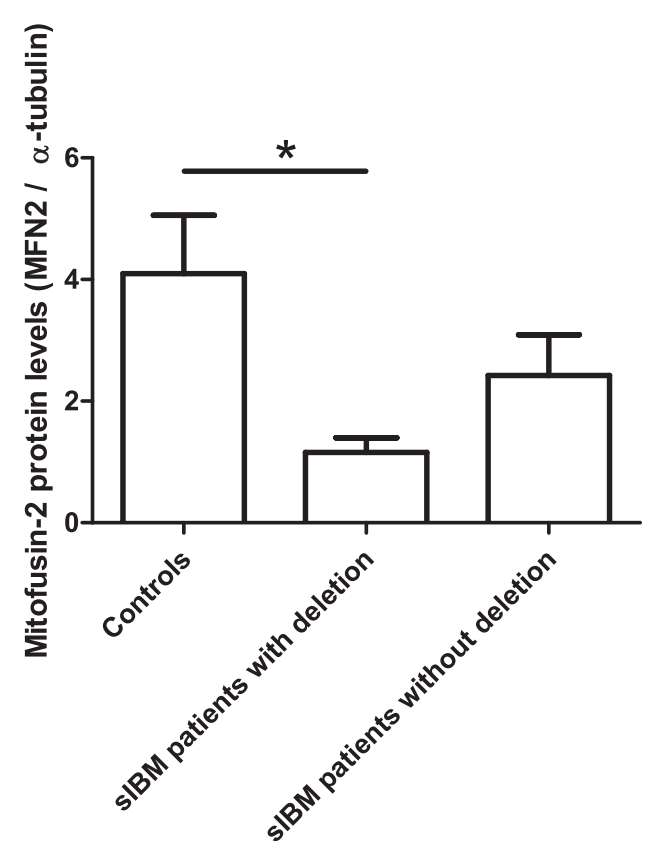

Figure 5 MtDNA amount and MFN2 protein levels in muscle of sIBM patients with and without mtDNA deletions and controls Comparison of (a) mtDNA amount and (b) MFN2 levels among patients with sIBM with and without mtDNA deletions and controls. The patients with sIBM and mtDNA deletions showed significantly reduced muscle levels of mtDNA and MFN2 protein levels with respect to the controls. The patients with sIBM and no mtDNA deletions presented an intermediate value, suggesting the implication of mtDNA deletions in other mitochondrial alterations or the involvement of mitochondrial dynamics in mtDNA homoeostasis. $*: p<0.05$.

mass, which additionally showed trends towards a decrease, according to poor homoeostatic compensation. Unlike in muscle, in PBMC nuclear-encoded protein subunits of COX (COX-IV) were slightly increased to compensate for COX dysfunction. The trends towards up-regulation of mitochondrially encoded proteins in muscle or nuclear-encoded proteins in PBMCs, to compensate for MRC dysfunction, may depend on the levels of endoplasmic reticular stress of both cell types, which has been reported to be altered in muscle $[39,40]$.

Mitochondrial dysfunction in sIBM patients is not caused by abnormal assembly of MRC complexes, at least as assessed through the measurement of protein levels of specific subunits that are labile in cases of complex instability. However, there was a strong trend of the complex $\mathrm{V}$ subunit, when normalized to mitochondrial mass, to be decreased. This decrease disappeared when the complex V subunit content was normalized to total cell protein. This could be explained by the slight increase in mitochondrial mass that underlies sIBM. Further analysis of complex $\mathrm{V}$ activity could be useful to elucidate whether this complex is altered in this disease.

Oxidative stress does not seem to play a role in SIBM pathogenesis, because lipid peroxidation levels are not increased in muscle or PBMCs from sIBM patients, at least in the present study cohort.

Overall, these data describe an altered mitochondrial phenotype in muscle and PBMCs of sIBM patients, with both tissues presenting a different lesion profile, probably due to different compensatory mechanisms related to the disease. It is interesting, regardless of the homoeostatic attempt to preserve mitochondrial function, that COX enzymatic deficiency is present in both tissues as a common pathogenic parallelism. Thus, it seems that mitochondrial dysfunction in sIBM is not confined only to the target tissue of the disease, muscle, but may also extend to other body tissues. Whether COX/CS activity could be used as a prognostic or severity biomarker of the disease should be assessed in further studies. Alternatively, PBMC mitochondrial alterations may be interpreted as a deregulation of the immune system, non-specific for sIBM, as stated before for other diseases with inflammatory components [41-45]. Regardless of the interpretation, mitochondrial impairment is present in both PBMCs and muscle tissue of sIBM patients.

Mitochondrial dynamics are the mechanism for this organelle recycling and turnover. Imbalance of proper ratios of fusion and fission events has recently been associated with disease $[13,46,47]$. Mitochondrial dynamics were severely altered in muscle of sIBM patients as demonstrated by the decreased expression of OPA1 and MFN2 transcript and protein levels. Deregulation of the mitochondrial fusion:fission ratio leads to abnormal mitochondrial turnover, which is essential for the recycling of damaged and dysfunctional mitochondria [48], such as those characteristic of sIBM patients.

It is interesting that reduced expression of MFN2 in sIBM patients is associated with abnormal mitochondrial genetics (both deletions and depletion). Previous studies have reported the strong dependence of mtDNA maintenance on proper mitochondrial dynamics in physiological conditions [10]. This is the first study to report this association in the context of SIBM disease.

Whether mitochondrial genetic and molecular alterations lead to abnormal mitochondrial dynamics or, on the contrary, to an 
imbalance in mitochondrial dynamics underlying the mitochondrial genetic and molecular disturbances is still a matter of debate. In any case, both adverse conditions seem to go hand in hand with the development of sIBM disease.

In summary, in the present study we demonstrate that mitochondria are impaired in sIBM, not only at histological but also at genetic, molecular and functional levels, confirming the involvement of mitochondria in the aetiology of this disease. The lesion is characterized by different patterns in both muscle and PBMCs of sIBM patients, demonstrating that mitochondrial injury is not exclusive to the target tissue of the disease but is also present in peripheral tissues. It is interesting that mitochondrial COX activity normalized by mitochondrial mass is altered in both tissues, highlighting the relevance of this parameter, and the need for further studies on this as a putative candidate diagnostic tool or therapeutic target. In addition, deregulation in mitochondrial dynamics seems to be crucial for mtDNA stability, not only in healthy conditions [10] but also in SIBM disease. Deregulation of mitochondrial dynamics in muscle leads to increased genetic alterations such as mtDNA depletion and multiple deletions, demonstrating that mitochondrial turnover is essential for proper mitochondrial health and, furthermore, for muscle function. Although further investigation is needed to demonstrate whether mitochondrial alterations are primary or secondary in SIBM, the present study provides more in-depth understanding of the role of mitochondria in this disease. It also provides knowledge related to the mechanisms implicated in order to help develop new diagnostic tools or therapeutic targets for this disease, in the absence of non-invasive diagnostic tools or effective therapy.

\section{CLINICAL PERSPECTIVES}

- The better understanding of how mitochondria is in SIBM would be useful at clinical level at different levels: to confirm that mitochondrial changes seen in the muscle biopsy of sIBM patients is a solid marker to help diagnose this disease.

- This information could be useful to develop new therapeutical strategies to treat muscle dysfunction in SIBM patients.

- The knowledge about the pathogenic mechanisms of the disease allow us to elaborate a more accurate description of the disease for the patient, as they could be able to know about the disease they're suffering.

\section{AUTHOR CONTRIBUTION}

J.M. Grau, along with F. Cardellach and G. Garrabou, conceived the study and supervised the data collection and analysis. J.M. Grau was responsible for the diagnosis and inclusion of all patients, performed the IBMFRS test and collected all the clinical data with the help of C. Morén. M. Catalán-García was responsible for the experimental analysis of the amounts of mtDNA and mtRNA (with the help of M. Bañó and J. Enrich-Bengoa), for the analysis of the MRC complex IV activity and CS activity (with the help of A. Hernando), for the analysis of the oxidative stress and mitochondrial protein synthesis (with the help of M. Guitart-Mampel and I. González-Casacuberta), and for the analysis of mitochondrial mass by Western blotting (with the help of D.-L. Juárez). MtDNA deletions were assessed by our collaborators S. Emperador and J. Montoya, and mitochondrial dynamics-related proteins were analysed through collaboration with A. Díaz-Ramos and A. Zorzano. E. Tobías provided technical assistance for reactive preparation. M. CatalánGarcía created a database to collect all clinical and experimental parameters and performed the statistical analysis of the data, under the supervision of G. Garrabou and C. Morén. All authors, especially G. Garrabou, J.M. Grau, J. Montoya and A. Zorzano, participated in the revision of the manuscript, adding new concepts of high relevance.

\section{ACKNOWLEDGEMENTS}

We would like to specially thank Fundació CELLEX for funding this project and to the extraordinary generosity of sIBM patients and families.

\section{FUNDING}

This study has been funded by Instituto de Salud Carlos III (ISCIII) [PI14/00005, PI15/00903, PI15/00817 and PIE1400061] and Fondo Europeo de Desarrollo Regional (FEDER), Suports a Grups de Recerca de la Generalitat de Catalunya [SGR 2014/376], La Marató [87/C/2015), Fundació CELLEX and CIBER of Rare Diseases (CIBERER, an initiative of ISCIII).

\section{REFERENCES}

1 Catalan, M., Selva-O'Callaghan, A. and Grau, J.M. (2014) Diagnosis and classification of sporadic inclusion body myositis (sIBM). Autoimmun. Rev. 13, 363-366 CrossRef PubMed

2 Needham, M., Corbett, A., Day, T, Christiansen, F., Fabian, V. and Mastaglia, F.L. (2008) Prevalence of sporadic inclusion body myositis and factors contributing to delayed diagnosis. J. Clin. Neurosci. 15, 1350-1353 CrossRef PubMed

3 Mastaglia, F.L. (2009) Sporadic inclusion body myositis: variability in prevalence and phenotype and influence of the MHC. Acta Myol 28, 66-71 PubMed

4 Vattemi, G., Mirabella, M., Guglielmi, V., Lucchini, M., Tomelleri, G., Ghirardello, A. and Doria, A. (2014) Muscle biopsy features of idiopathic inflammatory myopathies and differential diagnosis. Autoimmun. Highlights 5, 77-85 CrossRef

$5 \mathrm{Hu}$, J., Li, N., Yuan, J.H., Zhao, Z., Shen, H.R. and Mei, L. (2007) [A clinical and pathological analysis of inclusion body myositis. ] Zhonghua nei ke za zhi. 46, 658-660 PubMed

6 Joshi, PR., Vetterke, M., Hauburger, A., Tacik, P., Stoltenburg, G. and Hanisch, F. (2014) Functional relevance of mitochondrial abnormalities in sporadic inclusion body myositis. J. Clin. Neurosci. 21, 1959-1963 CrossRef PubMed

7 Rygiel, K.A., Miller, J., Grady, J.P., Rocha, M.C., Taylor, R.W. and Turnbull, D.M. (2014) Mitochondrial and inflammatory changes in sporadic inclusion body myositis. Neuropathol. Appl. Neurobiol. 41, 288-303 $\underline{\text { CrossRef }}$

8 Moslemi, A.R., Lindberg, C. and Oldfors, A. (1997) Analysis of multiple mitochondrial DNA deletions in inclusion body myositis. Hum. Mut. 10, 381-386 CrossRef

9 Mishra, P. and Chan, D.C. (2014) Mitochondrial dynamics and inheritance during cell division, development and disease. Nat. Rev. Mol. Cell Biol. 15, 634-646 CrossRef PubMed 
10 Chen, H., Vermulst, M., Wang, Y.E., Chomyn, A., Prolla, T.A., McCaffery, J.M. and Chan, D.C. (2010) Mitochondrial fusion is required for mtDNA stability in skeletal muscle and tolerance of mtDNA mutations. Cell 141, 280-289 CrossRef PubMed

11 Vielhaber, S., Debska-Vielhaber, G., Peeva, V., Schoeler, S., Kudin, A.P., Minin, I., Minin, I., Schreiber, S., Dengler, R., Kollewe, K. et al. (2013) Mitofusin 2 mutations affect mitochondrial function by mitochondrial DNA depletion. Acta Neuropathol. 125, 245-256 CrossRef PubMed

12 Chen, K.H., Dasgupta, A., Ding, J., Indig, F.E., Ghosh, P. and Longo, D.L. (2014) Role of mitofusin 2 (Mfn2) in controlling cellular proliferation. FASEB J 28, 382-394 CrossRef PubMed

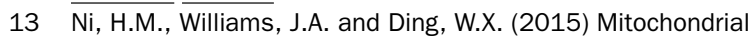
dynamics and mitochondrial quality control. Redox Biol. 4, 6-13 CrossRef PubMed

14 Dorn, G.W., 2nd, Vega, R.B. and Kelly, D.P. (2015) Mitochondrial biogenesis and dynamics in the developing and diseased heart. Genes Dev 29, 1981-1991 CrossRef PubMed

15 Guedes-Dias, P., Pinho, B.R., Soares, T.R., de Proenca, J., Duchen, M.R. and Oliveira, J.M. (2016) Mitochondrial dynamics and quality control in Huntington's disease. Neurobiol. Dis. 90, 51-57 CrossRef PubMed

16 Liesa, M., Palacin, M. and Zorzano, A. (2009) Mitochondrial dynamics in mammalian health and disease. Physiol. Rev. 89, 799-845 CrossRef PubMed

17 Gang, Q., Bettencourt, C., Machado, P., Hanna, M.G. and Houlden, H. (2014) Sporadic inclusion body myositis: the genetic contributions to the pathogenesis. Orphanet J. Rare Dis. 9, 88 CrossRef PubMed

18 Muller, F.L., Song, W., Jang, Y.C., Liu, Y., Sabia, M., Richardson, A. and Van Remmen, H. (2007) Denervation-induced skeletal muscle atrophy is associated with increased mitochondrial ROS production. Am. J. Physiol. Regul. Integr Compar. Physiol. 293, R1159-R1168 CrossRef

19 Sunitha, B., Gayathri, N., Kumar, M., Keshava Prasad, T.S., Nalini, A., Padmanabhan, B. and Srinivas Bharath, M.M. (2016) Muscle biopsies from human muscle diseases with myopathic pathology reveal common alterations in mitochondrial function. J. Neurochem. 138, 174-191 CrossRef PubMed

20 Sivakumar, K., Vasconcelos, O., Goldfarb, L. and Dalakas, M.C. (1996) Late-onset muscle weakness in partial phosphofructokinase deficiency: a unique myopathy with vacuoles, abnormal mitochondria, and absence of the common exon 5 /intron 5 junction point mutation. Neurology 46, 1337-1342 CrossRef PubMed

21 Moren, C., Garrabou, G., Noguera-Julian, A., Rovira, N., Catalan, M., Hernandez, S., Tobías, E., Cardellach, F., Fortuny, C. and Miró, Ò. (2013) Study of oxidative, enzymatic mitochondrial respiratory chain function and apoptosis in perinatally HIV-infected pediatric patients. Drug Chem. Toxicol. 36, 496-500 CrossRef PubMed

22 Garrabou, G., Hernandez, A.S., Catalan Garcia, M., Moren, C., Tobias, E., Cordoba, S., López, M., Figueras, F., Grau, J.M. and Cardellach, F. (2016) Molecular basis of reduced birth weight in smoking pregnant women: mitochondrial dysfunction and apoptosis. Addict. Biol. 21, 159-170 CrossRef PubMed

23 Liu, K., Sun, Y., Liu, D., Yin, J., Qiao, L., Shi, Y., Dong, Y., Li, N., Zhang, F. and Chen, D. (2013) Mitochondrial toxicity studied with the PBMC of children from the Chinese national pediatric highly active antiretroviral therapy cohort. PloS One 8, e57223 CrossRef PubMed

24 Houston, M.J. (2007) Muscle Biopsy. A practical approach. Elsevier 1, 416025936

25 Rose, M.R., ENMC IBM Working Group (2013) 188th ENMC International Workshop: Inclusion Body Myositis, 2-4 December 2011, Naarden, The Netherlands. Neuromuscul. Dis. 23, 1044-1055 CrossRef
26 Ascaso, F.J., Lopez-Gallardo, E., Del Prado, E., Ruiz-Pesini, E. and Montoya, J. (2010) Macular lesion resembling adult-onset vitelliform macular dystrophy in Kearns-Sayre syndrome with multiple mtDNA deletions. Clin. Exp. Ophthalmol. 38, 812-816 CrossRef

27 Carod-Artal, F.J., Lopez Gallardo, E., Solano, A., Dahmani, Y., Herrero, M.D. and Montoya, J. (2006) [Mitochondrial DNA deletions in Kearns-Sayre syndrome.]. Neurologia 21, 357-364 PubMed

28 Solano, A., Russo, G., Playan, A., Parisi, M., DiPietro, M., Scuderi, A., Palumbo, M., Renis, M., López-Pérez, M.J., Andreu, A.L. and Montoya, J. (2004) De Toni-Debre-Fanconi syndrome due to a palindrome-flanked deletion in mitochondrial DNA. Pediatr. Nephrol. 19, 790-793 CrossRef PubMed

29 Montero, R., Grazina, M., Lopez-Gallardo, E., Montoya, J., Briones, P., Navarro-Sastre, A., Land, J.M., Hargreaves, I.P., Artuch, R., Coenzyme $Q_{10}$ Deficiency Study Group (2013) Coenzyme $Q_{10}$ deficiency in mitochondrial DNA depletion syndromes. Mitochondrion 13, 337-341 CrossRef PubMed

30 Moren, C., Noguera-Julian, A., Garrabou, G., Rovira, N., Catalan, M., Bano, M., Guitart-Mampel, M., Tobías, E., Hernández, S., Cardellach, F. et al. (2015) Mitochondrial disturbances in HIV pregnancies. AIDS 29, 5-12 CrossRef PubMed

31 Moren, C., Noguera-Julian, A., Garrabou, G., Catalan, M., Rovira, N., Tobias, E., Cardellach, F., Miró, Ò. and Fortuny, C. (2012) Mitochondrial evolution in HIV-infected children receiving first- or second-generation nucleoside analogues. J. Acquir. Immune Defic. Syndr. 60, 111-116 CrossRef PubMed

32 Spinazzi, M., Casarin, A., Pertegato, V., Salviati, L. and Angelini, C. (2012) Assessment of mitochondrial respiratory chain enzymatic activities on tissues and cultured cells. Nat. Protoc. 7, 1235-1246 CrossRef PubMed

33 Garrabou, G., Moren, C., Lopez, S., Tobias, E., Cardellach, F., Miro, O. and Casademont, J. (2012) The effects of sepsis on mitochondria. J. Infect. Dis. 205, 392-400 CrossRef PubMed

34 Sebastian, D., Hernandez-Alvarez, M.I., Segales, J., Sorianello, E., Munoz, J.P., Sala, D., Waget, A., Liesa, M., Paz, J.C., Gopalacharyulu, P. et al. (2012) Mitofusin 2 (Mfn2) links mitochondrial and endoplasmic reticulum function with insulin signaling and is essential for normal glucose homeostasis. Proc. Natl. Acad. Sci. U.S.A. 109, 5523-5528 CrossRef PubMed

35 Hernandez-Alvarez, M.I., Thabit, H., Burns, N., Shah, S., Brema, I., Hatunic, M., Finucane, F., Liesa, M., Chiellini, C., Naon, D. et al. (2010) Subjects with early-onset type 2 diabetes show defective activation of the skeletal muscle PGC- $1 \alpha /$ Mitofusin-2 regulatory pathway in response to physical activity. Diabetes Care 33, 645-651 CrossRef PubMed

36 Oldfors, A., Moslemi, A.R., Jonasson, L., Ohlsson, M., Kollberg, G. and Lindberg, C. (2006) Mitochondrial abnormalities in inclusion-body myositis. Neurology 66 (2 Suppl 1), S49-S55 CrossRef PubMed

37 Herbst, A., Pak, J.W., McKenzie, D., Bua, E., Bassiouni, M. and Aiken, J.M. (2007) Accumulation of mitochondrial DNA deletion mutations in aged muscle fibers: evidence for a causal role in muscle fiber loss. J. Gerontol. A Biol. Sci. Med. Sci. 62, 235-245 CrossRef PubMed

38 Challa, S., Kanikannan, M.A., Murthy, J.M., Bhoompally, V.R. and Surath, M. (2004) Diagnosis of mitochondrial diseases: clinical and histological study of sixty patients with ragged red fibers. Neurol. India 52, 353-358 PubMed

39 Nogalska, A., D'Agostino, C., Terracciano, C., Engel, W.K. and Askanas, V. (2010) Impaired autophagy in sporadic inclusion-body myositis and in endoplasmic reticulum stress-provoked cultured human muscle fibers. Am. J. Pathol. 177, 1377-1387 CrossRef PubMed 
40 Nogalska, A., D’Agostino, C., Engel, W.K., Cacciottolo, M., Asada, S., Mori, K. and Askanas, V. (2015) Activation of the unfolded protein response in sporadic inclusion-body myositis but not in hereditary GNE inclusion-body myopathy. J.

Neuropathol. Exp. Neurol. 74, 538-546 CrossRef PubMed

41 Tezel, G., Fourth ARVO/Pfizer Ophthalmics Research Institute (2009) The role of glia, mitochondria, and the immune system in glaucoma. Invest. Ophthalmol. Vis. Sci. 50, 1001-1012 CrossRef PubMed

42 Lartigue, L. and Faustin, B. (2013) Mitochondria: metabolic regulators of innate immune responses to pathogens and cell stress. Int. J. Biochem. Cell Biol. 45, 2052-2056 CrossRef PubMed

43 West, A.P., Shadel, G.S. and Ghosh, S. (2011) Mitochondria in innate immune responses. Nat. Rev. Immunol. 11, 389-402 $\underline{\text { CrossRef PubMed }}$
44 Walker, M.A., Volpi, S., Sims, K.B., Walter, J.E. and Traggiai, E. (2014) Powering the immune system: mitochondria in immune function and deficiency. J. Immunol. Res. 2014, 164309 CrossRef PubMed

45 Gomez, L., Raisky, O., Chalabreysse, L., Verschelde, C., Bonnefoy-Berard, N. and Ovize, M. (2006) Link between immune cell infiltration and mitochondria-induced cardiomyocyte death during acute cardiac graft rejection. Am. J. Transpl. 6, 487-495 CrossRef

46 Santel, A. (2006) Get the balance right: mitofusins roles in health and disease. Biochim. Biophys. Acta 1763, 490-499 CrossRef PubMed

47 Burte, F., Carelli, V., Chinnery, P.F. and Yu-Wai-Man, P. (2015) Disturbed mitochondrial dynamics and neurodegenerative disorders. Nat. Rev. Neurol. 11, 11-24 CrossRef PubMed

48 Detmer, S.A. and Chan, D.C. (2007) Functions and dysfunctions of mitochondrial dynamics. Nat. Rev. Mol. Cell Biol. 8, 870-879 CrossRef PubMed 